\title{
Norois
}

Environnement, aménagement, société

$216 \mid 2010 / 3$

Territoire, Paysage, Anthropisation, Perception, Conservation, Restauration

\section{Définition d'unités paysagères par télédétection en Bretagne : méthodes et critiques}

Defining landscape units in Brittany by using remote sensing: methods and criticisms

\section{Émilie Bourget et Laurence le Dû-Blayo}

\section{(2) OpenEdition Journals}

\section{Édition électronique}

URL : http://journals.openedition.org/norois/3399

DOI : 10.4000/norois.3399

ISBN : 978-2-7535-1564-2

ISSN : $1760-8546$

Éditeur

Presses universitaires de Rennes

\section{Édition imprimée}

Date de publication : 15 novembre 2010

Pagination : 69-83

ISBN : 978-2-7535-1257-3

ISSN : 0029-182X

Référence électronique

Émilie Bourget et Laurence le Dû-Blayo, « Définition d'unités paysagères par télédétection en

Bretagne : méthodes et critiques », Norois [En ligne], 216 | 2010/3, mis en ligne le 01 décembre 2012, consulté le 30 avril 2019. URL : http://journals.openedition.org/norois/3399 ; DOI : 10.4000/ norois.3399 


\title{
Définition d’unités paysagères par télédétection en Bretagne : MÉTHODES ET CRITIQUES
}

\author{
Émilie Bourget, Laurence Le Dû-Blayo \\ Laboratoire COSTEL - UMR 6554 CNRS LETG \\ (Université Rennes 2 Haute-Bretagne), \\ Place du Recteur-Henri-Le-Moal, CS 24307 - 35000 Rennes \\ emilie.bourget@univ-rennes2.fr,laurence.ledu@univ-rennes2.fr
}

\section{RÉSUMÉ}

La gestion et la protection des paysages passe en premier lieu par leur connaissance, ainsi que le stipule la Convention Européenne du Paysage. Au sein des sources et des approches disponibles, la télédétection met à portée des utilisateurs des méthodes de définition d'unités paysagères pouvant être appliquées à différents espaces, et dont le principal intérêt réside dans une vision exhaustive des contrastes et transitions entre les territoires. Cependant, ces techniques reposant sur certains présupposés d'analyse des paysages, il convient d'étudier et comparer entre eux les différents résultats produits. Cet article présente une réflexion autour de trois traitements appliqués à une classification d’image satellite : une délimitation manuelle holistique, et deux traitements automatiques, l'un via le logiciel ClaPaS, et l'autre basé sur la morphologie des unités paysagères. L'objectif final est la cartographie des ensembles paysagers à l'échelle régionale au 1/250000, et l'harmonisation des démarches d'Atlas départementaux en cours ou futures.

MOTS CLÉ : paysages - unités paysagères - ensembles paysagers - télédétection - Bretagne

\section{ABSTRACT}

\section{Defining landscape units in Brittany by using remote sensing: methods and criticisms}

Landscapes management and protection can not be undertaken without a good knowledge about their characters, as it is stated in the European Landscape Convention. Remote sensing gives a wide range of methods to define landscapes units on different scales and areas. However, these techniques are based on some landscapes analysis presuppositions, so they give different results which have to be tested and compared. This article sets out a research which uses three processings applied on a satellite image classification: a manual, holistic delimitation, and two automatic processings, one via a software called ClaPaS, and the other based on the morphology of landscape units. The final purpose is the cartography of land units at the regional scale at 1:250000, and the harmonization of local landscape Atlases.

KEY WORDS : landscapes - landscape units - landscape groups - remote sensing Brittany 
Depuis près de quarante ans, la dégradation de certains paysages, tels le bocage ou les littoraux bretons, ont peu à peu provoqué chez les citoyens et les collectivités territoriales une prise de conscience et une volonté d'action qui tendent vers une gestion plus raisonnée du paysage, et de l'environnement en général. À l'échelle européenne, cette volonté s'est traduite par l'adoption par le Conseil de l'Europe, en 2000 à Florence, de la Convention Européenne du Paysage. Celle-ci définit le paysage comme " une partie de territoire telle que perçue par les populations, dont le caractère résulte de l'action de facteurs naturels et/ou humains et de leurs interrelations ». Pour être gérés et protégés, les paysages doivent d'abord être identifiés, et analysés, ainsi que le précise l'article 6 : chacun des États ayant signé la Convention s'engage notamment à « identifier ses propres paysages, sur l'ensemble de son territoire, à analyser leurs caractéristiques, [...] à qualifier les paysages identifiés ».

Cette nécessité de définition d'entités paysagères s'étend aux différentes échelles de réflexion et d'action. En France, les Atlas de Paysage tentent, depuis une quinzaine d'années, d'apporter différentes réponses à cette question, en inventoriant les paysages sous l'égide du ministère de l'Écologie, de l'Énergie, du Développement Durable et de la Mer, représenté par les DREAL (Directions Régionales de l'Environnement, de l'Aménagement et du Logement) (Luginbühl et al., 1994 ; Brunet-Vinck, 2004). À une échelle plus fine, les SCOT (Schémas de COhérence Territoriale) tentent également d'apporter des réponses en définissant des entités paysagères via des diagnostics paysagers effectués en amont des PADD (Projets d'Aménagement et de Développement Durable). Des études de paysage sont spécifiquement menées sur les Parcs Naturels Régionaux, qui ont notamment comme attribution, via leur charte, l'identification des types de paysages qui les constituent et la mise en œuvre de politiques paysagères (Loi Barnier de 1995). Les schémas éoliens contiennent également des analyses paysagères, à l'échelle régionale, départementale ou du Pays. Tous ces travaux ont en commun un but : celui d'identifier les paysages.

La loi Paysages de 1993 ainsi que différents séminaires inter-DIREN mis en place par le MEEDDM ont permis de préciser le vocabulaire et les échelles d'analyse des paysages, en distinguant les ensembles paysagers à l'échelle régionale (1/250 000), les unités de paysage (1/100 000), et les éléments de paysage (échelle locale); les structures paysagères, quelle que soit l'échelle, définissent les grands traits des paysages. Si l'idée d'un emboitement des échelles est partagé par toute la communauté scientifique, pour autant le vocabulaire n'est pas encore fixé; ainsi en Wallonie, les « ensembles paysagers » correspondent approximativement à l'échelle des « unités de paysages » en France (Van Eetvelde et Antrop, 2009). Ceux-ci sont subdivisés en territoires (1/50 000), eux-mêmes divisés en aires paysagères (1/20 000). Le terme d'entité sera ici utilisé comme terme générique pour toutes échelles confondues (Godart et Teller, 2007). Le présent travail a pour finalité de réaliser la synthèse des grands ensembles à l'échelle régionale, susceptibles de servir de trame à des cartographies plus fines d'unités de paysages. La mise en place de ces ensembles devra donc se faire en concertation avec les différents acteurs des politiques publiques d'inventaire des paysages.

Des méthodes d'identification des types et des unités paysagères existent, basées sur des critères paysagers objectifs et sur des perceptions du paysage plus subjectives; des solutions ont été présentées, à travers de nombreux Atlas des Paysages extrêmement variés par leur taille, leur contenu et leurs résultats. En Bretagne, plusieurs travaux ont déjà été menés soit à l'échelle régionale (DAT Conseil, 1995) soit à l'échelle départementale dans les Côtes-d'Armor et le Finistère, selon des méthodes différentes (Ouest Aménagement, 1995, 1996); mais le manque de cohérence dans les résultats n'a pas permis leur diffusion et leur prise en compte efficace dans les politiques publiques (Bligny, 2002). À l'échelle nationale, le SINP, sous l'égide du ministère de l'Écologie, de l'Énergie, du Développement Durable et de la Mer, mène un vaste programme afin de tenter une synthèse a posteriori des atlas déjà réalisés; de manière générale, le SINP prône un travail de concordance sur les zones frontalières lors de la mise en place des unités paysagères dans les Atlas des Paysages (séminaire SINP/DIREN de Tours, 2008). Un travail de comparaison entre les Atlas existants permet en effet d'observer une grande hétérogénéité des différentes publications et un manque de 
coordination entre les collectivités. Il en résulte des délimitations totalement dissemblables d'un territoire à l'autre et des entités paysagères limitrophes ne correspondant pas entre elles.

Des travaux similaires ont été menés dans la plupart des pays de l'Union Européenne (Wascher, 2005), comme en Espagne où le travail d'identification et de cartographie des paysages a été réalisé à l'échelle 1/200 000 (Mata Olmo et Sanza Herraiz, 2004). Cependant des difficultés semblables à celles rencontrées en France dans la concordance entre les découpages sur deux territoires voisins sont également observables chez certains de nos voisins européens. Ainsi en Belgique, la définition d'unités paysagères a été réalisée à une échelle et au moyen de techniques différentes en Flandres et en Wallonie, ce qui a produit des résultats trop hétérogènes pour pouvoir en l'état être mis en commun à l'échelle nationale (Van Eetvelde et Antrop, 2008, 2009).

L'usage de la télédétection, notamment à une échelle régionale, offre une vision globale rendant possible les comparaisons entre plusieurs espaces, et venant en complément des typologies réalisées à l'échelle européenne (Mücher et al., 2003), telle la classification LANMAP 2, qui reste insuffisante à l'échelle régionale. Plusieurs méthodes applicables à l'échelle d'une région ont ainsi été développées depuis une quinzaine d'années, offrant des résultats alternatifs aux unités paysagères vues au sens classique des Atlas de Paysage. On propose donc d'utiliser au mieux les contributions méthodologiques liées à la télédétection pour les adapter à l'identification des paysages et pallier aux problèmes de spatialisation et d'harmonisation inhérents aux approches terrain, et plus généralement à l'hétérogénéité des inventaires de paysages. L'objectif est donc multiple : il s'agit, in fine, de pouvoir proposer une spatialisation des ensembles paysagers par télédétection (et autres sources matricielles) qui puisse ainsi servir de trame à une harmonisation des Atlas en cours ou futurs.

\section{La définition des unités de paysage par télédétection}

\section{UNITÉS DE PAYSAGE ET TÉLÉDÉTECTION}

\section{Le concept d'unité de paysage depuis 1950}

L'unité de paysage est une notion présente dans divers champs de recherche depuis une soixantaine d'années. Elle a d'abord été développée en biogéographie à la fin des années 1960, quand G. Bertrand déclare que «le paysage est, sur une certaine portion d'espace, le résultat de la combinaison dynamique, donc instable, d'éléments physiques, biologiques et anthropiques qui, en réagissant dialectiquement les uns sur les autres, font du paysage un ensemble unique et indissociable » (Bertrand et Bertrand, 2002). On s'oriente vers des analyses holistiques où le paysage est vu comme un «tout » systémique et complexe, dépendant de facteurs biotiques, abiotiques et construits évoluant dans le temps (Zonneveld, 1989); c'est le Complexe Naturel Territorial (CNT) formalisé par la science du paysage soviétique et décrit par G. Rougerie et N. Beroutchachvili (1991). Cette vision globalisante entraîne de fait la nécessité d'un découpage de l'espace à une échelle adaptée, d'où l'émergence du concept d'unité de paysage (land unit) homogène. J. Wilmet (1981) insiste sur cet aspect d’homogénéité, qui ne se révèle « que par contraste avec l'environnement».

Ce type de travail prend son essor notamment à l'arrivée des premières séries de photographies aériennes, dans les années 1950. Le paysage, ainsi que l'unité de paysage à une échelle plus fine, sont vus comme des systèmes fonctionnant à plusieurs niveaux. Encore aujourd'hui, M. Martinelli (2007), sans utiliser la télédétection, adopte pour la Serra da Mantiqueira (Brésil), une méthode totalement systémique en associant intimement les divers éléments producteurs du paysage (géologie, pédologie, géomorphologie, végétation, urbanisation) lors de la définition des unités de paysages, qui sont vues ici comme des reflets des géosystèmes. J.-C. Wieber et al. (Brossard et Wieber, 2008) abondent dans ce sens lorsqu'ils insistent sur la nécessité de travailler dans une « optique affirmée [pour] traiter l'information de manière systématique et systémique ». À la fin des années 1970, la pédologie se saisit de ce concept d’unité homogène au travers du terme de 
pédopaysage, permettant d'extrapoler les formations de sols, par exemple à partir du modèle de croisement SCORPAN (Soil, Climate, Organisms, $\mathrm{R}=$ topography, Parental material, Age, $\mathrm{N}=$ space), qui établit des relations quantitatives entre les différentes variables afin de déterminer et d'extrapoler différents types de sols (McBratney et al., 2003; Le Dû-Blayo et al., 2008a).

Le développement des capteurs satellites depuis une quarantaine d'années a élargi les possibilités d'approche du paysage via la télédétection; dans le même temps, les préoccupations en faveur d'une gestion réfléchie et d'une préservation du cadre de vie font du paysage un objet d'étude à part entière, se détachant partiellement de la biogéographie et de l'écologie. Depuis les années 1990, les cartographies thématiques du paysage se multiplient, selon des méthodes très différenciées.

Parallèlement, le paysage devient l'objet de nombreuses mesures législatives ; ainsi, la loi « Paysages » du 8 janvier 1993 définit l'unité paysagère comme « un ensemble de composants spatiaux, de perceptions sociales et de dynamiques paysagères qui, par leurs caractères, procurent une singularité à la partie de territoire concernée ».

\section{La cartographie des entités paysagères}

La télédétection offre pour la définition des entités paysagères plusieurs avantages spécifiques. En effet, la variété des images proposées permet de travailler de manière multiscalaire (de l'Europe à la communauté de communes) en fonction de la résolution spatiale choisie (du kilomètre au mètre).

Il est intéressant de se pencher sur la réflexion de E. J. Gustafson (1998) à propos des représentations et de la cartographie du paysage. La donnée peut être ponctuelle et représenter le paysage par petites touches, à la manière des paysagistes. À l'inverse, la télédétection fournit via des classifications d'images satellite une information continue dans l'espace. De nombreuses recherches se situent entre ces deux extrêmes ou utilisent les deux possibilités, combinant des données ponctuelles souvent qualitatives sur la perception (analyse du terrain, représentations du paysage, données patrimoniales) à des données continues plus strictement descriptives (relief, occupation du sol, etc.).

La méthode développée en Belgique fait appel à une synthèse majoritairement quantitative réalisée à différentes échelles (Van Eetvelde et Antrop, 2008, 2009). Elle utilise quatre sources de données différenciées : un Modèle Numérique de Terrain (MNT), les données Corine Land Cover couvrant la Belgique, une carte des sols et une image satellite à haute résolution (Landsat 5 TM). La cartographie est ensuite mise en place selon deux étapes successives, d'abord en synthétisant les informations dans un carroyage d'1 km de côté, puis en regroupant les cellules du carroyage en unités paysagères.

Dans leur grande majorité, les différentes méthodes de caractérisation et de délimitation d'entités paysagères obéissent à une même philosophie dans leur mise en œuvre. Elles abordent la définition d'entités de paysages de manière systémique, à l'aide de plusieurs sources de données de nature diversifiée et selon deux grandes étapes :

- une première phase de croisement des données, qui aboutit à la définition des types de paysages présents et à une cartographie au pixel ;

- une seconde phase de synthèse des types de paysage, qui conduit à la délimitation des entités paysagères et à une cartographie vectorielle.

Les deux phases du traitement de données:

la typologie de paysages et la cartographie des entités paysagères

Différentes données peuvent être utilisées avec ces méthodes aboutissant aux typologies de paysages; cependant, leur degré divers de disponibilité conduit à retrouver le plus souvent le même type d’informations à la base des caractérisations des entités paysagères (tableau 1). 


\begin{tabular}{|l|c|c|}
\hline \multirow{2}{*}{ Information recherchée } & Données & Échelle/Résolution \\
\hline \multirow{3}{*}{ Occupation du sol } & Images satellite & Multiples \\
\cline { 2 - 3 } & Photos aériennes & $1 / 25000$ \\
\cline { 2 - 3 } & Corine Land Cover & $1 / 100000$ \\
\hline Végétation & Images satellite & Multiples \\
\hline Altitude/Pente & MNT & $50 \mathrm{~m}$ \\
\hline Géologie & Cartes géologiques & $1 / 50000$ \\
\hline Pédologie & Carte des sols & $1 / 100000$ \\
\hline Climat & Images satellite & Multiples \\
\hline Démographie/Économie & Statistiques & Multiples \\
\hline
\end{tabular}

Tableau 1 : Données numériques courantes utilisées dans les typologies de paysages Usual digital data used in landscape typologies

En fonction du nombre de sources de données retenues, le nombre de variables permettant d'aboutir à une typologie est changeant d'une méthode à l'autre. Bien entendu les taxons de la légende de la typologie de paysages seront également différents car ils sont en relation avec les sources utilisées, l'échelle de cartographie, le territoire étudié.

Ces variables sont ensuite croisées selon divers procédés, dont les plus usités sont (Van Eetvelde et Antrop, 2009) :

- l'overlay (superposition de plusieurs couches de données);

- la classification par cluster (regroupement des pixels en classes données à l'avance par l'utilisateur, et définies par les pixels les plus représentés).

Le choix des sources de données et du type de croisement par l'opérateur se fait en fonction de la connaissance du terrain et des types de paysages que l'on souhaite obtenir a priori. Le croisement de ces variables aboutit à différents types de paysages dont l'organisation spatiale va permettre de synthétiser des entités paysagères. Il semble ici primordial de traiter les données de manière spécifique pour une typologie adaptée à la différenciation des paysages et conçue en tant que telle.

\section{INTÉGRATION DES APPROCHES GÉOMATIQUES ET DES APPROCHES PAYSAGÈRES DE TERRAIN}

La méthodologie développée dans la présente recherche choisit de se baser sur une approche du paysage par télédétection (classification d'une série d'images MODIS et du MNT), tout en s'enrichissant, lors d'étapes ultérieures, des connaissances appartenant aux approches paysagères développées par les différents experts sollicités (bureaux d'étude, services de l'état, comités techniques, etc.) (fig. 1). Différents traitements sont ainsi appliqués à la classification, avant d'être synthétisés visuellement pour obtenir une première carte d'unités paysagères. Celle-ci est ensuite mise en commun et comparée avec les Atlas départementaux en construction, afin de produire une cartographie des ensembles paysagers régionaux en cohérence avec les données départementales.

Une base de données est construite en parallèle au processus, dans l'objectif de garder une trace des données ayant servi à construire la carte de synthèse. Cette base de données permet notamment de cartographier une typologie des limites des entités paysagères, en fonction de leur netteté et leur progressivité. Des données qualitatives (sites inscrits et classés, espaces naturels sensibles, etc.) pouvant enrichir la cartographie des entités paysagères viendront ultérieurement s’intégrer à la base de données, afin de préciser la description des ensembles paysagers. 


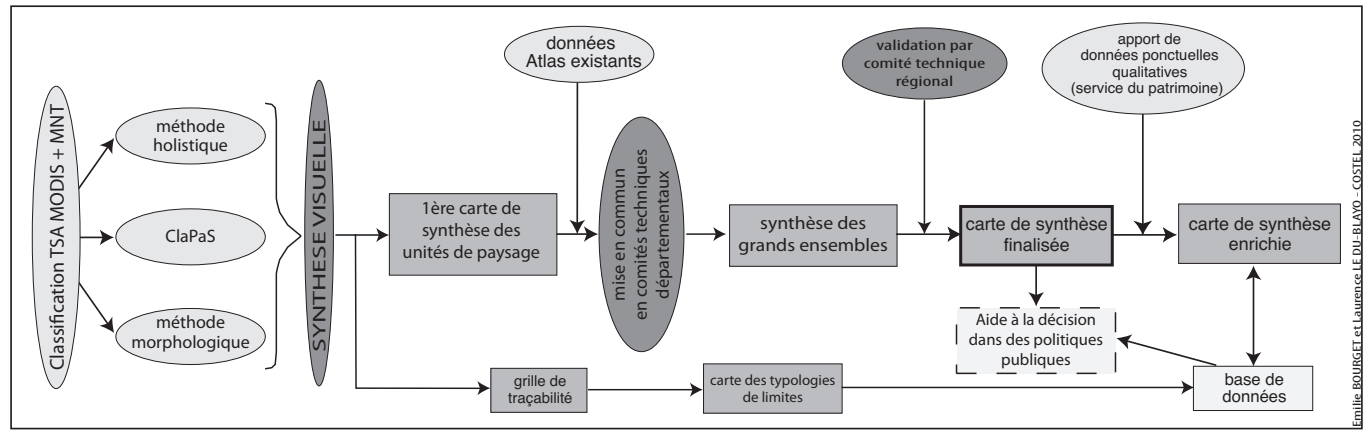

Figure 1 : Schéma de travail pour la définition d'ensembles paysagers en Bretagne Figure for the definition of landscape unities in Brittany

\section{Méthodologie expérimentée sur l'exemple breton}

\section{LA TYPOLOGIE DES PAYSAGES}

Pour cette expérimentation sur la Bretagne, la donnée de départ est constituée par la classification d'une série d'images MODIS (MODerate resolution Imaging Spectroradiometer, tableau 2) et des données de pentes et d'altitude du MNT de la Bretagne (résolution spatiale : $50 \mathrm{~m}$ ), réalisée en 2006 dans le cadre du programme IGCS - Sols de Bretagne conduit par l'INRA de Rennes (Michel et al., 2006; Le Dû-Blayo et al., 2008b). Sur le grand Ouest en effet, la classification Corine Land Cover ne permet pas de bien discriminer les paysages agraires, qui sont en très grande majorité compris dans une vaste classe « zones agricoles hétérogènes », d'où le choix de produire une typologie de paysages adaptée. La résolution spatiale du capteur MODIS, de $250 \mathrm{~m}$, le rend en très pertinent pour les études à l'échelle régionale, par la précision de l'information délivrée : chaque pixel couvre 6,25 ha et constitue donc une première synthèse des paysages observés; de plus la surface couverte n'oblige pas à des mosaïquages contraignants et source d'erreur. Par ailleurs, quatre images satellite prises à différentes saisons ont été analysées par TSA (Analyse de Séries Temporelles) afin de mieux cerner les contrastes paysagers au fil des années.

La typologie utilisée met en valeur les contrastes paysagers régionaux, qui sont renforcés par la prise en compte des reliefs (MNT). La carte des types de paysages en Bretagne ainsi produite constitue une première donnée spatialisée qui a été largement diffusée et exploitée par les acteurs privés et publics (GIP Bretagne Environnement, 2008).

\section{LES MÉTHODES DE REGROUPEMENT EN UNITÉS PAYSAGÈRES}

La deuxième phase de traitement consiste donc, à partir de la carte des types de paysages, à effectuer des regroupements en entités homogènes : unités puis ensembles. Plusieurs méthodes ont été mises en œuvre :

- Une recherche holistique sur les délimitations, réalisée visuellement, selon les connaissances préalables de l'opérateur (Le Dû-Blayo, 2007). Cette méthode constitue un premier choix facile et rapide à mettre en œuvre pour la définition d'unités paysagères par télédétection (Wilmet, 1981 ; Van Eetvelde et Antrop, 2009); elle peut servir de référence pour des comparaisons avec les résultats obtenus par les méthodes automatiques.

- Un traitement automatique effectué avec le logiciel ClaPaS (CLAssement de PAysages et Segmentation) (Robbez-Masson, 1994, 1999), qui permet d'obtenir des unités paysagères par agrégation de pixels, et dont l'utilisation se justifie par la similarité des objectifs entre son utilisation de départ sur les pédopaysages et celle qui en est faite dans la présente étude. 


\begin{tabular}{|l|l|}
\hline MODIS & 18 décembre 1999 \\
\hline Date de lancement & $705 \mathrm{~km}$ \\
\hline Altitude & $2330 \mathrm{~km}$ \\
\hline Fauchée & 1 à 2 jours \\
\hline Répétitivité & $250 \mathrm{~m}$ à $1 \mathrm{~km}$ \\
\hline Résolution spatiale & 36 bandes spectrales \\
\hline Bandes spectrales & 0,4 à $14,4 \mu \mathrm{m}$ \\
\hline Radiométrie & B : $0,459-0,479 \mu \mathrm{m}$ \\
\hline \multirow{2}{*}{ dont: } & $\mathrm{V}: 0,545-0,565 \mu \mathrm{m}$ \\
\cline { 2 - 2 } & $\mathrm{R}: 0,620-0,670 \mu \mathrm{m}$ \\
\cline { 2 - 2 } & $\operatorname{PIR}: 0,841-0,876 \mu \mathrm{m}$ \\
\cline { 2 - 2 } & $\operatorname{MIR}(1): 1,230-1,250 \mu \mathrm{m}$ \\
\cline { 2 - 2 } & $\operatorname{MIR}(2): 1,628-1,652 \mu \mathrm{m}$ \\
\cline { 2 - 2 } & $\operatorname{MIR}(3): 2,105-2,155 \mu \mathrm{m}$ \\
\hline
\end{tabular}

Tableau 2 : Principales caractéristiques techniques du capteur MODIS Main technical features of MODIS sensor

- Un deuxième traitement automatique, basé lui sur la morphologie des entités paysagères (Le Dû-Blayo et al., 2008a, 2008b), plus expérimental et venant en complément des deux premiers traitements; il permet également d'obtenir des unités paysagères par agrégation de pixels.

Les trois approches méthodologiques pour l'extraction des unités de paysage sont basées sur la même classification.

\section{Une délimitation visuelle et holistique des unités paysagères}

Cette méthode issue de la photo-interprétation est la plus classique et a été mise en œuvre dès les années 1970 par de nombreux géographes (Breyelle et Wilmet, 1975; Coudoux, 1986), car elle répond bien à une recherche de synthèse territoriale simple sur des critères multiples complexes (types de paysages, structures paysagères, hétérogénéité, etc.). En effet, celui qui interprète l'image va opérer des regroupements sur des combinaisons difficilement modélisables : parfois le contraste spectral sera prédominant, d'autres fois le relief, ou une organisation spatiale particulière. La méthode a donc l'avantage de traiter rapidement des informations complexes, et l'inconvénient de ne pas être strictement reproductible à l'identique. La définition d'entités paysagères sur la classification MODIS a été effectuée en 2007 par L. Le Dû-Blayo pour le programme IGCS Sols de Bretagne (Le Dû-Blayo et al., 2008b; Le Dû-Blayo, 2007). Ce travail visuel constitue une synthèse des diverses informations contenues dans l'image (type de paysage, altitude, pente), et a été facilité par une connaissance étendue du terrain d'étude (géologie, relief, flore, agriculture, etc.), qui a permis de nuancer le type de limite existant entre les unités paysagères (limite marquée ou limite progressive).

\section{Une définition automatique des unités paysagères avec le logiciel ClaPaS}

Le logiciel ClaPaS (CLAssement de PAysages et Segmentation) a été développé en 1994 par J.-M. Robbez-Masson à l'ENSA de Montpellier dans une optique de définition d'unités de pédopaysages. ClaPaS « a pour objectif de réaliser le classement d'une image sur la base de la composition paysagère du voisinage de chacune de ses cellules »(Robbez-Masson, 1994, 1999). ClaPaS permet de déterminer à quel paysage de référence rattacher chaque pixel de l'image, en fonction de la composition de son voisinage spatial. Des études ont montré la possibilité de 
caractériser des paysages en utilisant l'outil ClaPaS (Bornand et al., 1997), bien que celui-ci ait été élaboré pour l'étude des sols.

L’utilisation du logiciel débute par la définition de «paysages de référence » sur la classification d'image satellitale qui va être traitée. Ceux-ci sont décrits par un histogramme de composition et une matrice de co-occurrences qui donne la distribution des variables dans chaque échantillon de référence considéré.

Après différents essais, 26 zones de référence d'une taille de $30 \times 30$ pixels ou $2 \times(15 \times 30)$ pixels (soit 5625 ha) ont été définies (fig. 2 - planche IV), représentant en partie la diversité des types de paysages qu'on peut rencontrer en Bretagne.

Puis le module Clapas opère le classement de chaque pixel de l'image d'après la distance mathématique entre le descripteur des échantillons de référence (leurs histogrammes de composition), et le descripteur de chaque pixel et son voisinage; ce classement se fait au sein d'une fenêtre de taille et de forme variables.

Les précédentes études de la Bretagne utilisant ClaPaS et le capteur MODIS (Michel, 2006; Vergne, 2006) tendent à montrer qu'il est préférable d'adopter certaines options de calcul adaptées à l'hétérogénéité des paysages bretons. Ainsi, il a été décidé de réaliser différents essais sur la classification MODIS avec les options suivantes :

- des voisinages de taille maximale $\mathrm{T}_{\max }$ la plus grande possible, pour obtenir un résultat satisfaisant à l'échelle des unités paysagères;

- une forme de voisinage ronde;

- des paramètres mathématiques de description paysagère les mieux adaptés : l'histogramme comme descripteur, et la distance de Manhattan comme type de distance mathématique (Bornand et al., 1997; Lagacherie et al., 2001).

Un filtre modal de taille 7x7 pixels est ensuite appliqué à l'image afin de lisser l'information obtenue.

Une définition automatique des unités paysagères basée sur la morphologie mathématique

Un post-traitement basé sur la morphologie mathématique des unités de paysage a été développé par P. Gouéry en interne à l'Université Rennes 2 en 2006 (Le Dû-Blayo et al., 2008a). Il répond à une volonté de conserver lors du post-traitement les particularités de certaines des classes paysagères, ce qu'il n'est pas possible de faire avec d'autres méthodes plus classiques (application de filtres, etc.), notamment ClaPaS. La méthode de ce post-traitement consiste à traiter séparément, au moyen de processus de morphologie mathématique, chacune des classes de paysages, et ce de manière itérative pour faire successivement varier les paramètres des opérateurs mathématiques ; une synthèse est ensuite faite de toutes les données récoltées pour chaque classe de paysage, en tenant compte des possibilités de recouvrement affectées ou non à chacune des classes. Ce nouveau post-traitement permet de prendre en compte la taille et la forme des différentes classes de paysages de la classification MODIS. En effet, sur la classification MODIS les différentes classes de paysage dessinent des zones très différentes les unes des autres tant par leur forme que par leur taille, et chaque classe a une signification qu'il est plus ou moins opportun d'agréger. De plus, les surfaces de grande taille (telles celles de bocage dense) sont susceptibles d'inclure en leur sein de petites zones d'autres classes de paysage, telle une zone boisée ou urbaine, qu'il peut être intéressant de conserver dans le post-traitement.

Le respect de la diversité paysagère par ce post-traitement nécessite en effet de spécifier quelles classes sont prioritaires sur les autres lors du recouvrement, et si elles sont mixables entre elles ou non. Ainsi, certaines classes de paysages apparaissant par petites zones sont déclarées prioritaires et non mixables, afin qu'elles ne disparaissent pas lors du post-traitement; c'est le cas notamment des zones de paysage urbain, des zones boisées, etc. À l'inverse, les classes de paysages qui apparaissent sur des surfaces étendues (tels les bocages, les paysages mixtes, les plateaux et bassins cultivés à paysage ouvert), peuvent être recouvertes par un paysage prioritaire, ou mixées entre elles pour donner naissance à de nouvelles classes de paysages. 


\section{Resultats}

\section{Les résultats de la délimitation visuelle et holistique}

Il résulte de cette recherche un découpage en 62 unités de la péninsule bretonne (fig. 3 planche V), unités qui sont en cohérence avec les résultats présentés dans l'Atlas des Paysages de Basse-Normandie. Elles sont de taille et de forme différentes, parfois petites telle l'unité de la cuvette de Porzay ( $n^{\circ} 43$, fig. 3 - planche V), parfois beaucoup plus étendues comme l'unité des Crêtes de Bain de Bretagne ( ${ }^{\circ} 58$, fig. 3 - planche V); cependant elles constituent des ensembles fermés et relativement homogènes. Cette première carte, publiée en 2007, est exploitée dans différentes études paysagères à des échelles plus fines (schéma territorial éolien 2008, Syndicat Mixte du Pays de Saint-Malo). Ce travail constitue une base de réflexion pour la présente recherche, que viendront enrichir et pondérer les résultats des deux traitements automatiques en vue d'une synthèse.

\section{Les résultats avec le logiciel ClaPaS}

En sortie, ClaPaS fournit trois images différentes : une image du résultat du classement, une image de la distance mathématique entre les descripteurs des paysages de référence et celui de chaque voisinage de pixel, et enfin une image présentant la taille de voisinage optimale, comprise entre $\mathrm{T}_{\min }$ et $\mathrm{T}_{\max }$, retenue pour le traitement de chaque pixel.

On obtient une représentation des unités de paysages bretons (fig. 4 - planche VI), avec une très grande diversité de taille et de forme des zones, qui sont de taille moyenne et de forme « fermée » sur l'ouest de la région (Monts d'Arrée, Montagnes Noires, plateaux du Trégor et du Léon), tandis que l'est est occupé par des unités plus diffuses, en nappes (Bassin de Rennes, Bassin de Pontivy-Loudéac, partie avale de la Vilaine, côte Nord). Malgré une bonne agrégation des types de paysage, certaines unités sont trop petites, et ces « confettis» ne sont pas représentatifs à l'échelle régionale. $\mathrm{ClaPaS}$ est adapté à des nuances de pédopaysages fines mais dans des paysages très hétérogènes, le regroupement en unités de grande surface est plus délicat.

\section{Les résultats avec le traitement basé sur la morphologie}

Le résultat du post-traitement utilisant la morphologie mathématique (fig. 5 - planche VII) fait apparaître des paysages plus morcelés, d'avantage présents sous forme de petites, voire de très petites unités, que dans le post-traitement effectué avec le logiciel ClaPaS, notamment dans le bassin de Rennes et sur la côte sud de la Bretagne. Il met d'avantage en avant certaines nuances (Penthièvre) et également la spécificité des zones mixtes (2 types de paysages également représentés sur une surface donnée).

\section{SyNTHÈSE ET CARTOGRAPHIE DES UNITÉS DE PAYSAGE}

On voit sur les trois traitements que les mêmes grandes tendances se dégagent visiblement, notamment sur l'ouest de la région, avec la bonne individualisation des deux massifs des Monts d'Arrée et des Montagnes Noires, des plateaux du Léon et du Trégor; le même phénomène se retrouve à l'est sur les frontières de la Normandie, où le bocage s'individualise également très bien. À l'inverse, les paysages mixtes de la Cornouaille, du Goëlo et de tout le sud-est de la Bretagne sont moins aisément agrégés en unités.

Les deux derniers post-traitements testés lors de l'étude ne produisent pas des unités paysagères au sens classique des Atlas de Paysage, compte tenu de l'hétérogénéité des paysages bretons, mais ils délivrent une information supplémentaire en vue d'une synthèse, notamment sur l'organisation des types de paysage. Alors que le premier post-traitement fournit de grandes unités plus ou moins homogènes, on obtient avec les deux autres des résultats beaucoup plus morcelés, avec des tailles d'unités inférieures à celles souhaitées pour un Atlas. C'est pourquoi le choix d'une synthèse visuelle des trois post-traitements a été mis en œuvre (fig. 6 - planche VIII), dont il 
résulte 97 unités de paysage sur la Bretagne, de taille et de forme très variées, intéressantes pour travailler à l'échelle départementale.

Cette synthèse a été exécutée selon la technique des «calques numériques », mise au point à l'INRA-SAS dans le cadre du programme IGCS - Sols de Bretagne, et qui permet de superposer les différentes unités, facilitant la prise de décision de l'opérateur (Berthier et al., 2009) pour effectuer la synthèse visuelle des trois traitements.

Cette méthode des calques numériques permet de prendre en compte la traçabilité dans la construction des limites d'unités de paysage, avec une base de données spécifique. L'opérateur indique dans la table attributaire quelles informations ont permis la délimitation de l'unité, et quelle est leur part d'importance dans la prise de décision. Chaque polygone d'unité de sol définie est subdivisé en autant de polylignes que de type de choix, et chacune des polylignes constitue une ligne dans la base de données, où il est précisé quelles variables ont permis sa définition.

Cette phase de synthèse permet de combiner les informations issues des trois méthodes et d'extraire une donnée pouvant ensuite être croisée avec celle des Atlas en cours ou réalisés.

\section{Confrontation avec les Atlas}

La comparaison entre la classification des images satellite MODIS, et les différents Atlas en cours de construction en Bretagne (sur les départements du Finistère, du Morbihan et de la LoireAtlantique) permet une complémentarité de l'analyse, entre l'échelle régionale de la classification et l'échelle départementale des Atlas (fig. 7 - planche VIII). L'échelle régionale offre en effet une première vue harmonisée des ensembles paysagers, complétée par l'analyse plus fine donnée par les Atlas départementaux, qui s'appuient notamment sur une analyse terrain approfondie et également sur une dimension de perception des paysages, absentes de la classification d'images satellite MODIS. La complémentarité de ces deux approches permet par la suite une synthèse en grands ensembles paysagers régionaux.

\section{SYNTHÈSE FINALISÉE EN ENSEMBLES PAYSAGERS RÉGIONAUX}

Comme souligné précédemment, l'échelle régionale nécessite de travailler avec de grands ensembles, les unités de paysage départementales étant inadaptées à ce niveau. Cette synthèse (fig. 8 - planche IX - recherche réalisée avec le soutien du conseil régional de Bretagne) a été réalisée en collaboration avec les équipes chargées de la réalisation ou de l'actualisation des atlas départementaux (bureaux d'étude privés, services territoriaux de l'État) sur trois départements (Morbihan, Finistère et Loire-Atlantique), et en tenant compte des unités frontalières de l'atlas des paysages de la Basse-Normandie (Brunet et Girardin, 2003). On aboutit à la délimitation de 39 ensembles paysagers, cohérents au niveau régional et permettant une première approche du paysage à cette échelle. Cette synthèse est en cours de validation par un comité de pilotage régional qui regroupe les différents acteurs (État, Région, Conseils Généraux, CAUE, etc.).

\section{Discussion et perspectives}

\section{LA PRISE EN COMPTE DE L'INCERTITUDE DANS LES RÉSULTATS}

Dans les méthodes testées ici, l'incertitude du classement et de la délimitation des entités apparaît sous plusieurs formes, mais n'est pas forcément explicitée. L'incertitude peut se situer à trois niveaux différents de l'analyse (Corgne, 2004) :

- incertitude liée à l'exactitude des données ;

- incertitude liée à la méthode de traitement des données;

- incertitude liée au résultat. 
Dans la méthode visuelle holistique, l'incertitude relève surtout de la méthode, toujours empirique, mais paradoxalement les résultats sont satisfaisants par rapport à l'objectif d'entités paysagères synthétiques. L'incertitude apparaît dans les différents types de limites : limite «marquée » ou limite « progressive », cette dernière s'apparentant davantage pour l'expert à la transition progressive, au continuum paysager qu'au changement franc entre deux entités.

Le logiciel ClaPaS exprime lui l'incertitude liée au résultat, à savoir la difficulté à classer certains pixels, dans une carte représentant la distance mathématique entre les paysages de référence et le voisinage de chaque pixel. Plus la distance mathématique est importante, plus l'appartenance du pixel à une unité paysagère est incertaine (fig. 9 - planche $\mathbf{X}$ ).

Si on exclut le littoral, qui mixe au sein de chaque fenêtre des pixels très divers, les zones des paysages de référence n’ont eu évidemment aucune difficulté à être bien identifiées et classées (distances faibles, représentées en bleu très foncé); inversement les zones plus claires de la carte (distance importante, en vert et jaune) sont celles que le logiciel considère comme ayant été les plus difficiles à classer, du fait de grandes distances mathématiques entre leur composition et la composition des paysages de référence; le résultat sera donc plus incertain dans ces zones (fig. 10 - planche $\mathbf{X}$ ). Il s'agit de zones assez spécifiques, comme les Landes de Lanvaux, les bordures de la Forêt de Paimpont ou le Trégor (pointées par les flèches rouges), trois cas très différents :

- les Landes de Lanvaux sont difficiles à classer par le logiciel du fait d'un effet de structure laniérée du paysage, qui est composite, car lié notamment à la structure géologique et aux altitudes (relief appalachien);

- la Forêt de Paimpont est une unité offrant une frontière marquée, constituée de pixels composites, avec les autres unités de paysages l'entourant;

- le Trégor est une région au paysage morcelé et mixte, avec de très petites zones de différents types de paysages, les unes à côté des autres; le logiciel a également des difficultés à agréger ce type de zones, très fréquent en Bretagne.

Ces exemples permettent de mieux visualiser les différents cas d'incertitude pouvant survenir lors de la phase de délimitation des unités paysagères.

La synthèse visuelle effectuée plus haut avec les calques numériques permet également de prendre en compte l'incertitude des limites (fig. 11 - planche XI). En effet, dans la table de données accompagnant cette synthèse, un coefficient de 0 (incertitude faible) à 3 (incertitude forte) a été attribué par l'opérateur pour qualifier l'incertitude dans la construction les limites de synthèse :

On remarque une incertitude faible sur les paysages de l'ouest et de l'extrême est de la Région (prioritairement, des paysages «fermés » de type bocage ou des plateaux légumiers), qui se retrouvent de manière quasi-identique dans les différents traitements. À l'inverse, on retrouve une forte incertitude sur le sud et l'est de la zone étudiée (paysages avant tout mixtes et ouverts). La traçabilité des données permet de savoir quel(s) résultat(s) de traitements ont permis de mettre en place ces limites d'unités de paysage. Les modes de cartographie des trois méthodes étudiées peuvent être résumées comme suit (tableau 3).

\begin{tabular}{|l|c|c|c|}
\hline \multirow{2}{*}{ Méthodes } & \multicolumn{3}{|c|}{ Cartographie des entités } \\
\cline { 2 - 4 } & Type de synthèse & $\begin{array}{c}\text { Traçabilité des don- } \\
\text { nées }\end{array}$ & Incertitude \\
\hline À dire d'expert & Visuelle & Non & Qualitative \\
\hline ClaPaS & Automatique & Non & Quantitative \\
\hline Morphologie & Automatique & Partielle & Non \\
\hline
\end{tabular}

Tableau 3 : Caractéristiques de cartographie des trois traitements Processing features of the three processings 
Il est nécessaire de souligner qu'aucune des trois méthodes de post-traitement testées ici n'est entièrement satisfaisante. Elles impliquent toutes les trois pour l'opérateur de faire des choix lors de leur mise en place, que ce soit lors de la délimitation des unités de paysage dans la méthode à dire d'expert, ou lors du choix des options de calcul et d'échantillonnages dans le cas des méthodes automatiques. Lors de leur synthèse visuelle, l'opérateur sera également amené à faire des choix dans sa manière de synthétiser les différentes informations. On peut cependant s'accorder sur le fait que chacun des post-traitements présente un apport pour certaines agrégations en unités et que leur synthèse offre un enrichissement pour l'extraction des ensembles à l'échelle régionale.

\section{EXPORTATION DE STATISTIQUES SUR LES ENTITÉS ET ENRICHISSEMENT PAR DES DONNÉES EXOGÈNES}

Des statistiques peuvent être produites pour décrire quantitativement les entités paysagères, par les types de paysages qui les composent, afin d'obtenir une typologie de ces entités et de connaître leurs types de paysage dominants. Ce travail a notamment été réalisé sur les 62 unités paysagères définies visuellement et a été inclus dans les réflexions du CSEB (Conseil Scientifique de l'Environnement de Bretagne) dans le cadre d'une saisine sur le bocage en 2007 (Bretagne Environnement - CSEB, 2009).

Les entités paysagères peuvent être dans un second temps décrites à une échelle plus fine avec des données qualitatives par commune, comme l'inventaire des monuments historiques, le patrimoine naturel relevé dans des guides touristiques ou les zones protégées au titre de diverses conventions (Ramsar, Natura 2000, etc.). L'objectif est ici de compléter la qualification des grands ensembles paysagers par leur valeur patrimoniale.

L'ensemble de paysage est un zonage susceptible d'être croisé de manière constructive avec des données dynamiques détaillées telles que le RGA (Recensement Général Agricole); les tendances ne sont probablement pas les mêmes pour le nombre d'exploitants agricoles ou l'évolution de la $\mathrm{SAU}$, dans une unité de paysage à tendance bocagère et dans une unité dominée par un paysage de plaine ouverte. Ce type de croisement est informatif, notamment pour des prospectives à l'échelle du paysage.

Ces différents exemples de l'exploitation potentielle de l'unité ou de l'ensemble de paysage en tant qu'entité de réflexion et d'action montrent l'intérêt qu'il y aurait à l'intégrer, avec toutes les statistiques qui y sont rattachées, dans le processus de certaines politiques publiques.

\section{EXPORTATION DE LA MÉTHODOLOGIE SUR D'AUTRES TERRITOIRES}

La méthode expérimentée sur la Bretagne a permis d'aboutir aux deux objectifs visés :

- d'une part la délimitation des entités paysagères sur la base de données numériques (télédétection et relief);

- d'autre part, sur la base de cette expertise, l'harmonisation, à l'échelle régionale, entre les Atlas départementaux via les ensembles paysagers.

Ainsi, les données exhaustives régionales par télédétection sont utilisées en complémentarité des expertises terrain des paysagistes afin d'éviter les incohérences interdépartementales, mais également d'optimiser la spatialisation des paysages (vision du dessus) et leur définition fine (approche terrain). La télédétection apporte une certaine vision du paysage, venant enrichir une approche in situ plus conventionnelle.

Le déroulement général de la procédure a été ici mis en œuvre de manière expérimentale en fonction des acquis (programme IGCS, Le Dû-Blayo et al., 2008b), des méthodes de traitement disponibles (visuelles, ClaPaS, morphologie mathématique) et des Atlas en cours (Finistère, Morbihan, Loire-Atlantique). On aboutit à un résultat cohérent qui articule les échelles régionale et départementale, les approches numérique et paysagère, les expertises scientifiques et les arbitrages des acteurs. 
De fait, il en ressort non pas une méthode automatique universelle, ce qui n'est pas envisageable sur le sujet, mais une démarche pragmatique (fig. 12) qui peut être reproduite sur d'autres territoires en s'adaptant aux données et aux acteurs disponibles. Le facteur clé de la réussite des cartographies d'ensembles à l'échelle régionale réside dans la qualité de l’identification des types de paysages en amont.

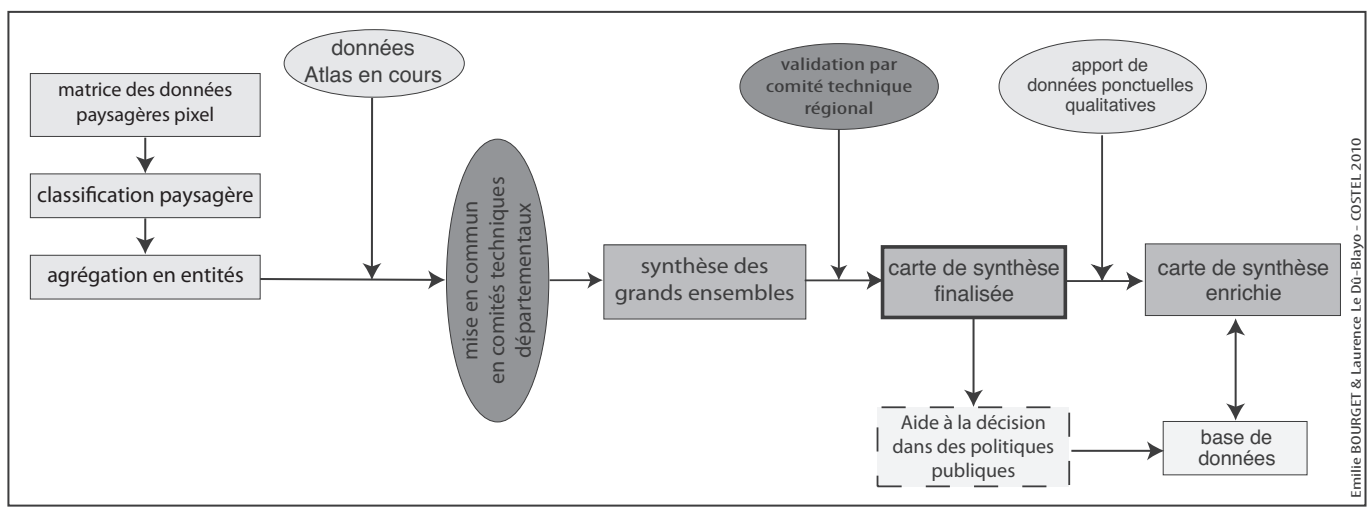

Figure 12 : Schéma de travail général pour la définition d'ensembles paysagers régionaux General figure for the definition of regional landscape unities

\section{Conclusion}

Un des enjeux principaux de cette recherche est la connaissance et la délimitation des différents types de paysages présents sur un territoire donné. La télédétection offre, parmi de nombreuses approches possibles, plusieurs méthodes permettant de définir des entités paysagères. Les tests menés sur le territoire de la Bretagne ont démontré la complexité de la répartition spatiale des types de paysage et donc la difficulté à les regrouper en unités puis en grands ensembles paysagers. De manière pragmatique, c'est la combinaison de méthodes automatiques et de méthodes holistiques (travail de synthèse visuelle, apport de connaissance experte) qui offre les résultats les plus satisfaisants. En concertation avec les différents acteurs du paysage, elle permet à terme d'aboutir à une synthèse d'ensembles paysagers à l'échelle régionale, qui vise à harmoniser les travaux des différents Atlas paysagers départementaux. Les cartographies obtenues doivent également rentrer dans un processus de validation par les acteurs publics afin d'être effectivement exploitées dans la mise en œuvre des politiques publiques. De fait, c'est la cohérence partagée de ces inventaires paysagers qui ouvre sur une exploitation effective dans la mise en œuvre des politiques sectorielles (schéma éolien, schéma bocager, Trames Vertes et Bleues, etc.), celles-ci étant alors adaptées aux spécificités des entités paysagères. Enfin, on envisage aussi d'enrichir la connaissance de chaque entité paysagère par des données statistiques quantitatives, et par des données qualitatives à échelle fine. Il faut souligner que cette méthodologie, sans être « exportable » telle quelle, est reproductible et applicable sur d'autres régions, pouvant à terme rendre possible une certaine harmonisation de la donnée, sinon sur le territoire national, du moins sur le Grand Ouest. 


\section{Bibliographie}

Antrop M., Van Eetvelde (V.), 2005. The implementation of the landscape atlas of Flanders in the integrated spatial planning policy, Landscape and urban planning, vol. 70, nº 1-2, p. 127-141.

Bligny C., 2002. Bilan des atlas des paysages en France, Rapport ENGREF, Clermont-Ferrand, 129 p.

Berthier L., Le Bris A.-L., Lemercier B., Walter C., 2009. Démarche qualité et cartographie régionale des sols dans le cadre du programme Sols de Bretagne, Actes des 10 Journées d'Étude des Sols, Strasbourg, 11-15 Mai 2009, non paginé.

Bertrand C., Bertrand G., 2002. Une géographie traversière - L'environnement à travers territoires et temporalités, Paris, Quae, $311 \mathrm{p}$.

Bornand M., Robbez-Masson J.-M., Donnet A., Lacaze B., 1997. Caractérisation des sols et paysages des garrigues méditerranéennes - Typologie et extrapolation spatiale par traitement d'images satellitaires, Étude et gestion des sols, $\mathrm{n}^{\circ}$ 4/1, p. 27-43.

Bou Kheir R., Girard M.-C., Khawlie M., 2004. Utilisation d'une classification structurale OASIS pour la cartographie d'unités de paysages dans une région représentative du Liban, Journal Canadien de Télédétection, vol 30, $\mathrm{n}^{\circ} 4$, p. 617-630.

Bourget E., Le Dû-Blayo L., 2008. Mapping Landscape identities at regional scale - The case of Brittany (France), Communication, $23^{\mathrm{e}}$ session de la PECSRL - Landscapes, identities and development, Lisbonne/ Obidos, Portugal, 1-5 septembre 2008.

Bretagne Environnement, CSEB, 2009. Contribution sur la question du bocage en Bretagne, Rapport d'étude, $71 \mathrm{p}$. [http://www.csebbretagne.fr/index.php?option=com_remository\&Itemid=28\&func $=$ select Eid=26] (février 2010).

Breyelle P., Wilmet J., 1975. Étude régionale d'une partie de l'Europe du Nord-Ouest sur composition colorée obtenue à partir de données Landsat 1, Photo-interprétation, n 6, p. 26-41.

Brunet P., Girardin P., 2004. Inventaire régional des paysages de Basse-Normandie, Rapport DIREN BasseNormandie/Conseil Régional Basse-Normandie, 2 tomes, 323 p et 871 p. [http://www.basse-normandie. ecologie.gouv.fr/Inventaire.html] (mai 2010).

Brunet-Vinck V., 2004. Méthode pour des Atlas de paysages - Enseignements méthodologiques de 10 ans de travaux, Rapport final, Bureau des Paysages - ministère de l'Écologie et du Développement, Paris, 48 p.

Conseil de L’Europe, 2000. Convention Européenne du Paysage, Florence, 7 p.

Congne S., 2004. Modélisation prédictive de l'occupation des sols en contexte agricole intensif : application à la couverture hivernale des sols en Bretagne, Thèse de Doctorat, Université Rennes 2 - Haute-Bretagne, Rennes, $250 \mathrm{p}$.

Coudoux J., 1986. Zonage par téléanalyse et Petites Régions Agricoles (PRA) de la région Nord-Pas-deCalais, Hommes et Terres du Nord, $\mathrm{n}^{\circ}$ 1, p. 64-72.

DAT ConsEIL, 1995. Réflexions pour une politique régionale sur les paysages en Bretagne, Rapport DIREN Bretagne et Conseil Régional de Bretagne, 63 p.

Gip Bretagne Environnement, 2008. L'environnement en Bretagne : cartes et chiffres clés, Rennes, 164 p.

Girard M.-C., Mougenot B, Ranaivoson A., 1991. Présentation d'un modèle d'organisation et d'analyse de la structure des informations spatialisées: OASIS, dans Caractérisation et suivi des milieux terrestres en régions arides et tropicales, Bondy, Éditions ORSTOM, p. 341-350.

Godart M.-F., Teller J. (dir.), 2007. Atlas des paysages de Wallonie: 1. L'Entre-Vesdre-et-Meuse, Namur, Publication CPDT, 263 p.

Godart M.-F., Feltz C. (dir.), 2009. Atlas des paysages de Wallonie : 2. Les plateaux brabançon et hesbignon, Namur, Publication CPDT, 283 p.

Gustafson E. J., 1998. Quantifying landscape spatial pattern : What is the state of art?, Ecosystems, vol. 1, p. 143-156.

Lagacherie P., Robbez-Masson J.-M., Nguyen-The N., Barthes J.-P., 2001. Mapping of reference area representativity using a mathematical soilscape distance, Geoderma n 101, p. 105-118.

Le Dû-Blayo L., 2007. Le paysage en Bretagne - enjeux et défis, Plomelin, Éditions Palantines, 350 p. 
Le Dû-Blayo L., Gouery P., Corpetti T., Michel K., Lemercier B., Walter C., 2008a. Improving the input of remotely-sensed data and information into digital soil maps, dans HarTeminck A. E. et al., Digital soil mapping with limited soil data, Éditions Elsevier, Coll. Developments in soil science series, p. 337-348.

Le Dû-Blayo L., Bourget E., Corpetti T., Gouery P., Michel K., 2008b. Esquisse cartographique des pédopaysages de Bretagne par télédétection - Rapport final, Programme Sols de Bretagne, 92 p.

LuginbüHl Y., Bontron J.-C., Cros Z., 1994. Méthode pour des atlas de paysages - identification et qualification, Paris-La Défense, Éditions Strates/CNRS-SEGESA, 76 p.

Martinelli M., 2007. The cartography of landscape units in São Bento do Sapucai - Serra da Mantiqueira (São Paulo, Brazil), Environmental Science and Engineering, Éditions Springer, p. 343-357.

Mata Olmo R., Sanza Herraiz C., 2004. Atlas de los paisajes de Espana, Madrid, Ministerio de Medio Ambiante, $683 \mathrm{p}$.

McBratney A. B., Mendonca Santos M. L., Minasny B., 2003. On digital soil mapping, Geoderma, n 117 , p. 3-52.

Michel K., 2006. Cartographie des pédopaysages de la Bretagne à l'aide de la télédétection, Rapport d'activité, Programme Sols de Bretagne, $54 \mathrm{p}$.

Mücher C. A., Bunce R. G. H., Jongman R. H. G., Klijne J. A., Koomen A. J. M., Metzger M. J., Wascher D. M., 2003. Identification and characterisation of environments and landscapes in Europe, Wageningen, Alterra, rapport 382, 119 p.

Ouest Aménagement, 1996. Diagnostic paysager sur le territoire départemental - Côtes-d'Armor, Rapport Conseil Général des Côtes-d'Armor, 136 p.

Ouest Aménagement, 1995. Inventaire et typologie des paysages du Finistère, Rapport Direction Départementale de l'Équipement du Finistère, 127 p.

Robbez-Masson J.-M., 1994. Reconnaissance et délimitation de motifs d'organisation spatiale - Application à la cartographie de pédopaysages, Thèse de l'Ecole Nationale Supérieure Agronomique de Montpellier, 161 p.

Robbez-Masson J.-M., Foltete J.-C., Cabello L., Flitti M., 1999. Prise en compte du contexte spatial dans l'instrumentation de la notion de paysage - Application à une segmentation géographique assistée, Revue internationale de géomatique, vol. 9, n² 2, p. 173-195.

Rougerie G., Beroutchachvili N., 1991. Géosystèmes et paysages : bilan et méthodes, Paris, Armand-Colin, coll. «U Géographie », 302 p.

SINP, 2008. Atelier sur les données paysages du SINP avec les DIREN Centre, Poitou-Charentes et Maine-etLoire, note interne, Tours, 20-21 mars 2008, 9 p.

Van Eetvelde V., Antrop M., 2009. A stepwise multi-scaled landscape typology and characterization for transregional integration, applied on the federal state of Belgium, Landscape and urban planning, $\mathrm{n}^{\circ}$ 91, p. 160-170.

Van Eetvelde V., Antrop M., 2008. A method for a multi-scale landscape typology and characterisation in a transregional context useful as a GIS-landscape database - Case of Belgium, Communication, $23^{\mathrm{e}}$ session de la PECSRL - "Landscapes, identities and development", Lisbonne/Obidos, Portugal, 1-5 septembre 2008.

Van Eetvelde V., Antrop M., 2004. Towards landscape characterization of Belgium - A new typology, Communication, ELCAI, Evora, 2-4 juin 2004.

VERGNe O., 2006. Comparaisons de méthodes de cartographies des limites paysagères floues par télédétection : application au Pays de Pontivy, Mémoire de Master 2 Géographie, Université de Rennes 2 - Haute-Bretagne, 37 p.

WASCHER D. M., 2005. European landscape character areas - typologies, cartography and indicators for the assessment of sustainable landscapes, Landscape Europe, 148 p.

Wieber J.-C. Griselin M., Ormaux S., 2008. Le paysage, objet de recherche scientifique? dans Brossard T. et Wieber J.-C., Paysage et information géographique, Paris, Éditions Hermès-Lavoisier, coll. « Information géographique et Aménagement du territoire », 414 p.

Wilmet J., 1981. Télédétection par satellite et espaces régionaux, L’Espace Géographique, n² 2-1981, p. 89-98.

Zonneveld I. S., 1989. The land unit - A fundamental concept in landscape ecology, and its applications, Landscape ecology, vol. 3, $\mathrm{n}^{\circ}$ 2, p. 67-86.

Cet article a été reçu le 3 mars 2009 et définitivement accepté le 9 juin 2010. 
planche IV (Guy-Serge BIGNOUMBA - Les pêches maritimes en Afrique centrale...)

(Émilie BOURGET, Laurence LE DÛ-BLAYO - Définition d'unités paysagères par télédétection...)

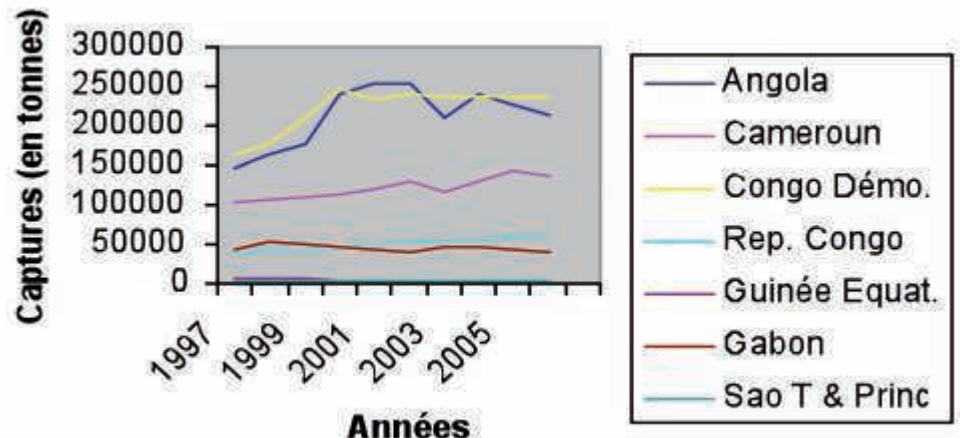

Figure 2 : Évolution des captures en Afrique centrale, de 1997 à 2006 (Source : FAO, captures par pays ou zones, 2006)

Evolution of captures in Central Africa, from 1997 till 2006

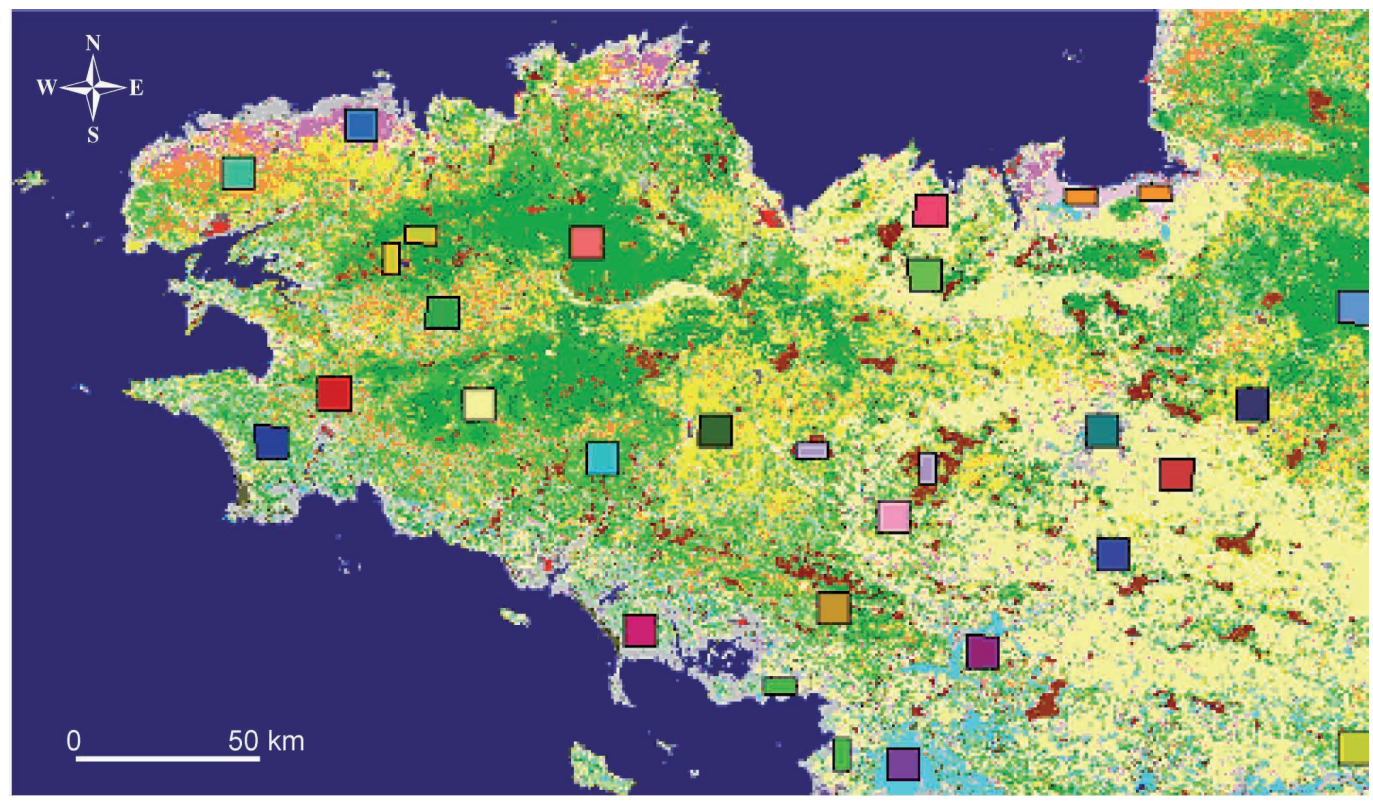

Figure 2 : Localisation des 26 zones de référence pour l'utilisation de ClaPaS (Bourget dans Le Dû-Blayo et al., $2008 \mathrm{~b})$

Location map of the 26 reference areas in ClaPaS using (Bourget in Le Dî-Blayo et al., 2008b) 


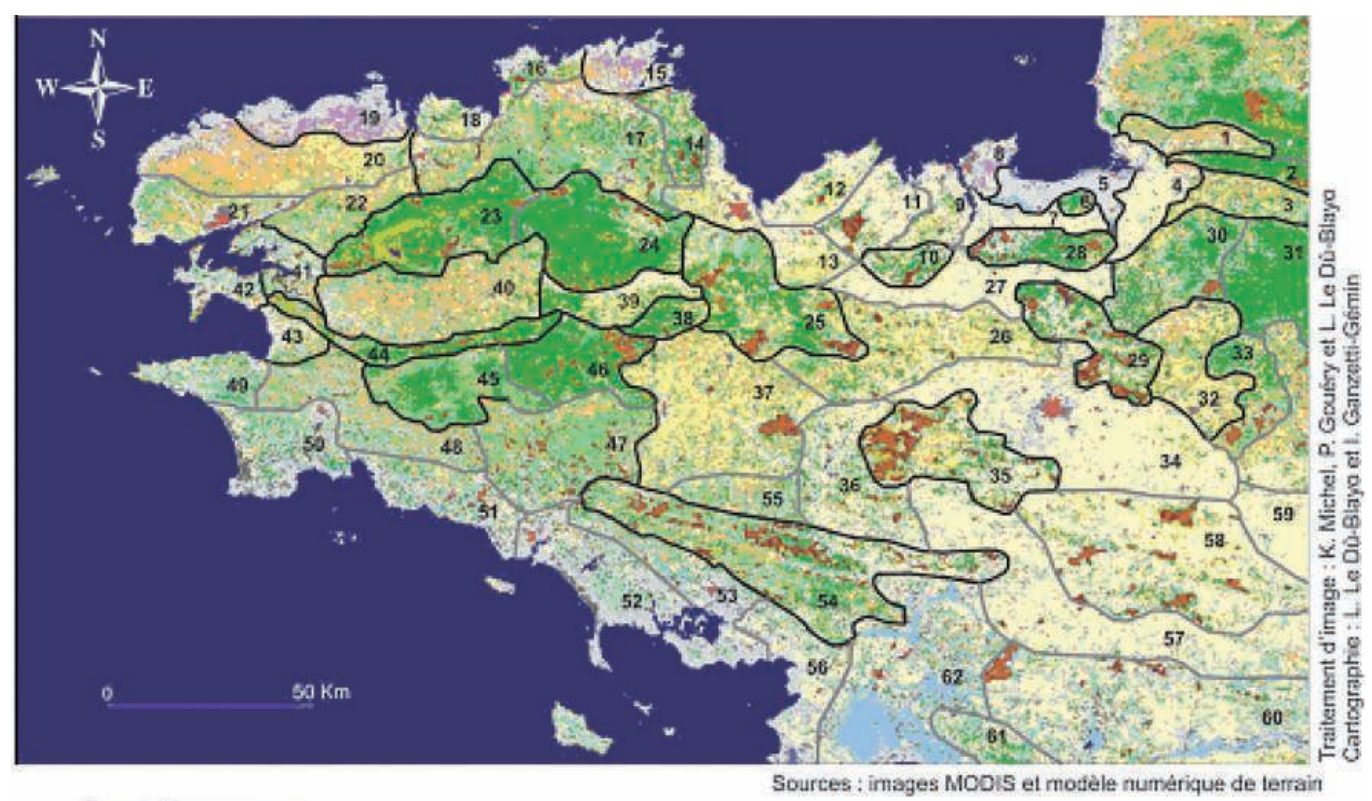

Types de paysages

Bocage dense et praifies sur collines Bocage à maille èlargie Paysages mixtes avec bosquets Plateaux avec paysages ouwerts of bocage relictuel Plateeux avec talus ou haies besses

Plaines avec bocage à ragosee destructuré Cultures de legumes sur plateaux Cultures de légumes sur plaines Forets a dominante de feuilus Focêts à dominante de résineux
Friches et landes

Marsis d'eau douce et zones humices

Mares fittoraux

Urbain dense

Periurbain et bourgs

Habilat tiffus

Dunes

Herbus

Mer, lacs, cours dfeau, estran

\section{Unités de paysages}

Figure 3 : Définition des unités de paysage par méthode holistique (L. Le Dû-Blayo, 2007) Definition of landscape units by holistic methodology (L. Le Dî-Blayo, 2007) 


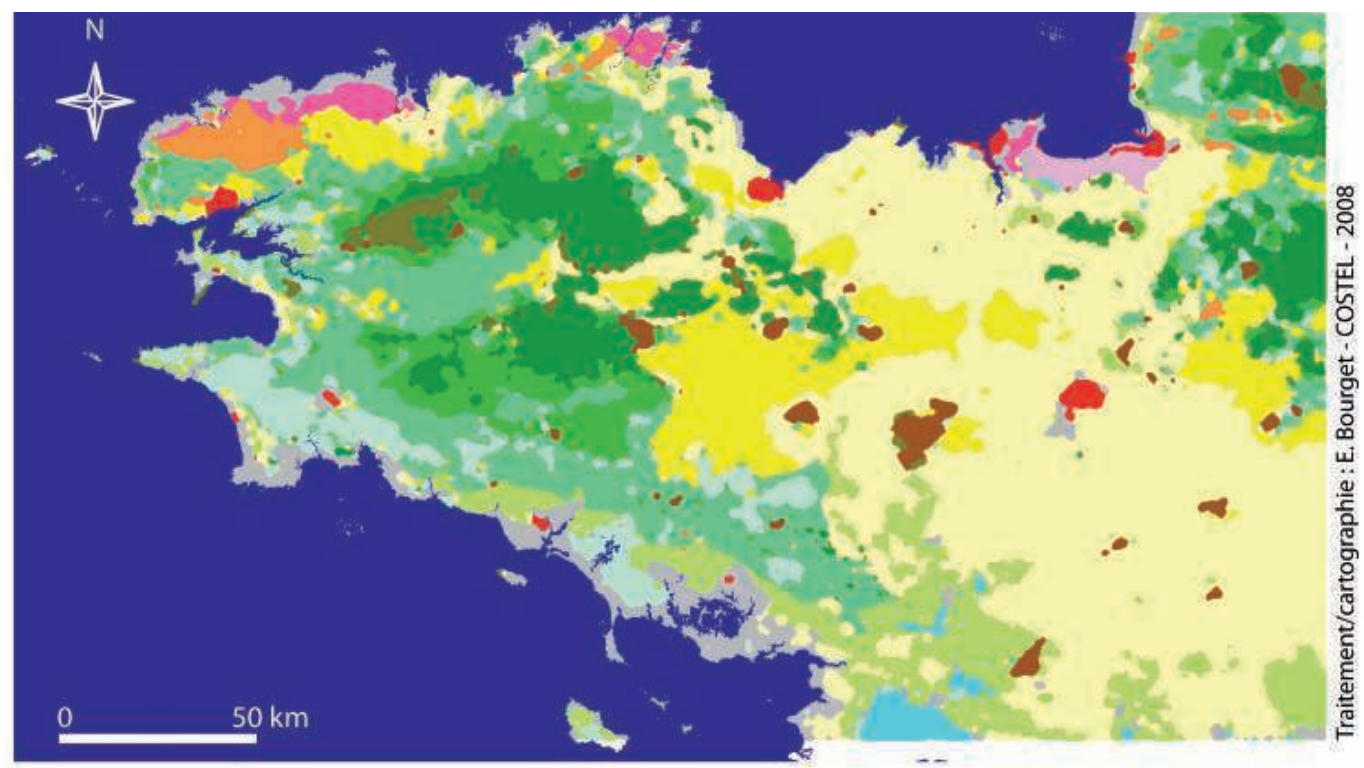

\section{Unités de paysage :}

Bocage dense des hauteurs de l'Argoat

Bocage élargi du piémont des massifs

Paysage mixte entre Armor et Argoat

Bocage élargi du littoral cornouaillais

Plateaux côtiers avec talus

Plateaux à paysages ouverts mixtes

Plateaux légumiers de la côte Nord

Plaine légumière du Marais de Dol
Plaines de Haute-Bretagne à bocage relictuel

Paysage mixte des plaines du Sud-Est

Landes et friches

Massifs forestiers de l'Argoat

Paysages urbains

Paysages périurbains ou de mitage littoral

Zones humides des Marais de Brière et Redon

Mer, lacs, cours d'eau

Figure 4 : Carte des types de paysages obtenus avec le logiciel ClaPaS (Bourget dans Le Dû-Blayo et al., 2008b) Map of landscape types obtained with ClaPaS software (Bourget in Le Dî-Blayo et al., 2008b) 


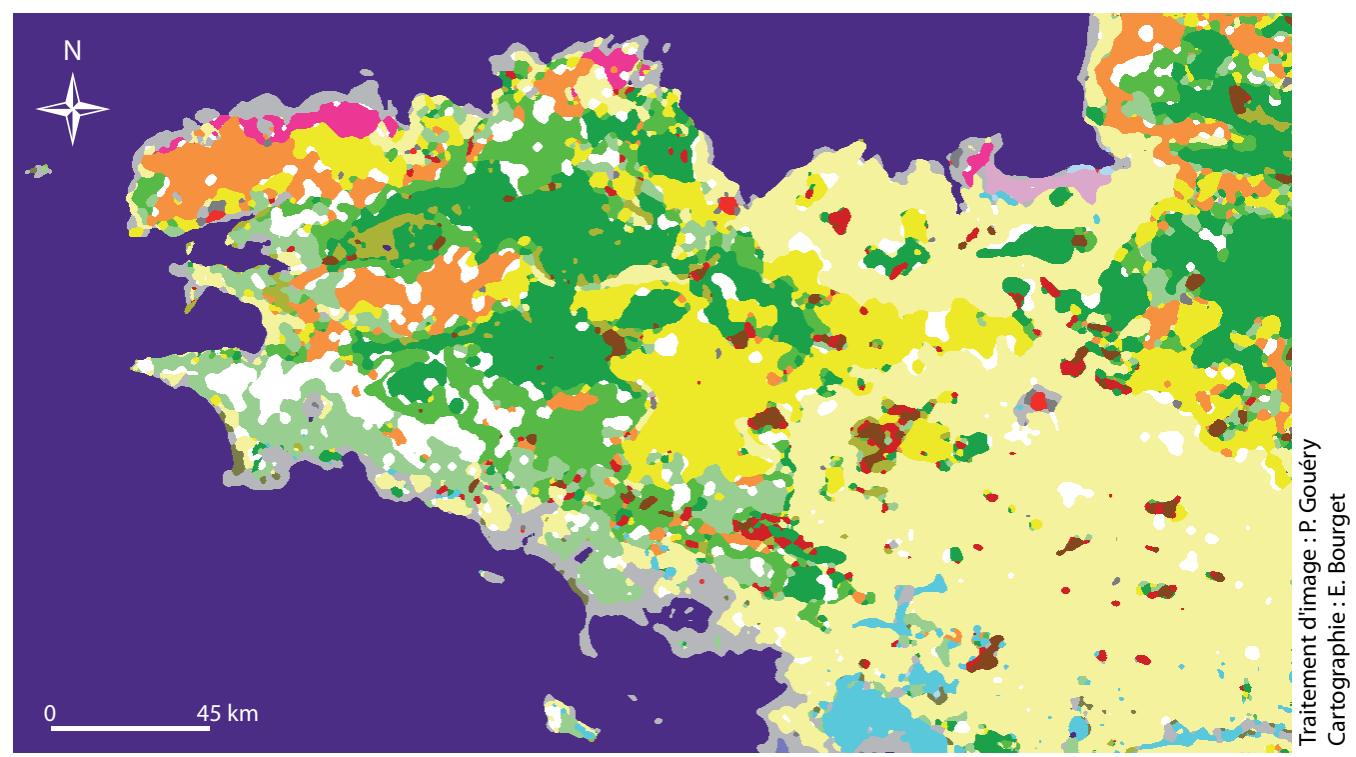

\section{Types de paysages}

Bocage dense et prairies sur collines

Bocage à maille élargie

Paysage mixte avec bosquets

Plateaux avec paysages ouverts et bocage relictue

Plateaux avec talus ou haies basses

Plaines avec bocage à ragosses destructuré

Cultures de légumes sur plateaux

Cultures de légumes sur plaines

Forêts à dominante de feuillus

Forêts à dominante de résineux
Friches et landes

Marais d'eau douce et zones humides

Marais littoraux

Urbain dense

Périurbain et bourgs

Habitat diffus

Dunes

Herbus

Mer, lacs, cours d'eau, estran

Zones de paysages mixés

Figure 5 : Carte des types de paysage obtenus par traitement morphologique (Gouéry dans Le Dû-Blayo et al., 2008b)

$2008 b)$

Map of landscape types obtained with the morphological processing (Gonéry in Le D̂̂-Blayo et al., 


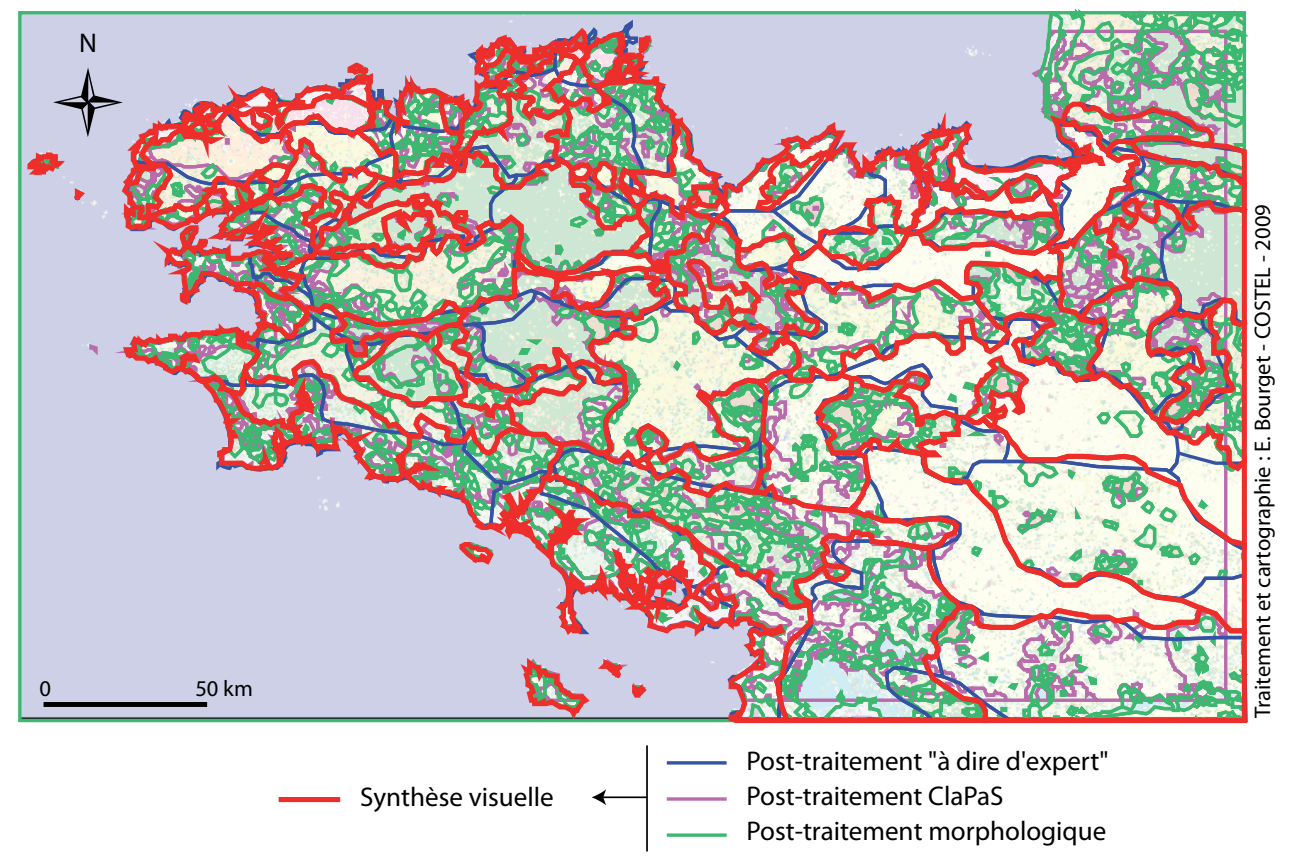

Figure 6 : Délimitation d'unités de paysage par synthèse visuelle des trois traitements Delimitation of landscape units by visual synthesis of the three processings

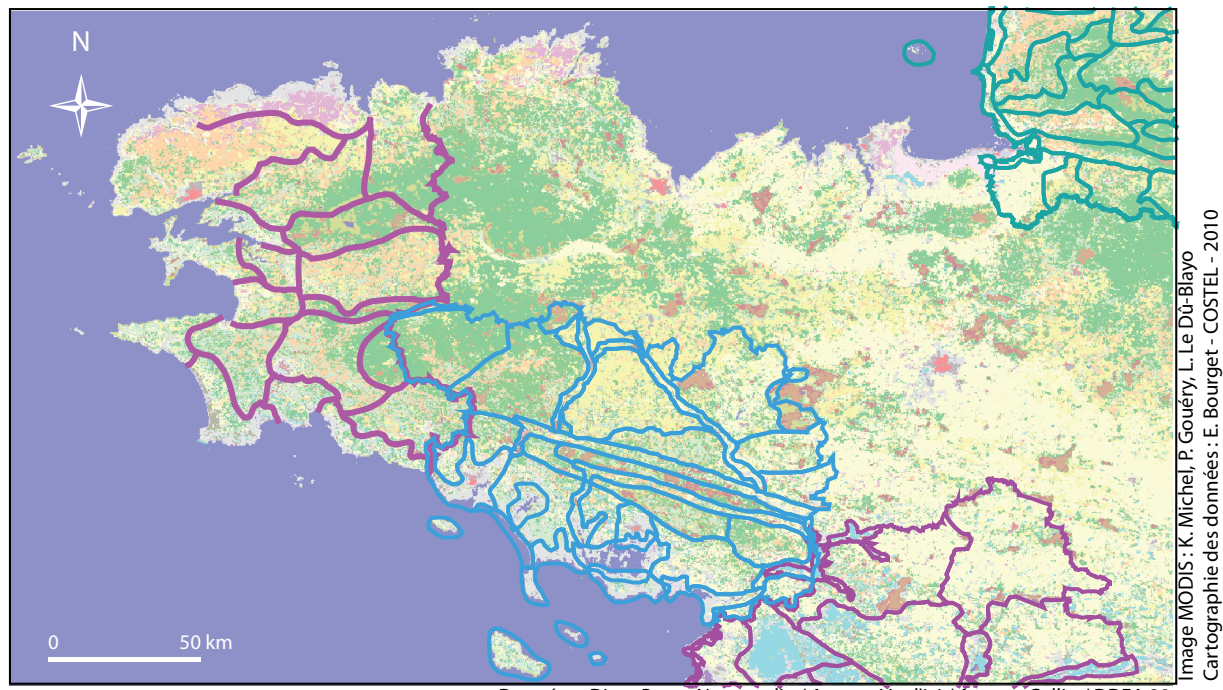

Données : Diren Basse-Normandie / Agence Vu d'ici / Ágence Collin / DDEA 29

Figure 7 : Comparaison entre les données satellite et les données des Atlas de paysage

Comparison between remotesensed data and Landscape Atlases data
Atlas des Paysages de Basse-Normandie

Atlas des Paysages du Finistère

Atlas des Paysages du Morbihan (en cours)

Atlas des Paysages de Loire-Atlasntique (en cours) 


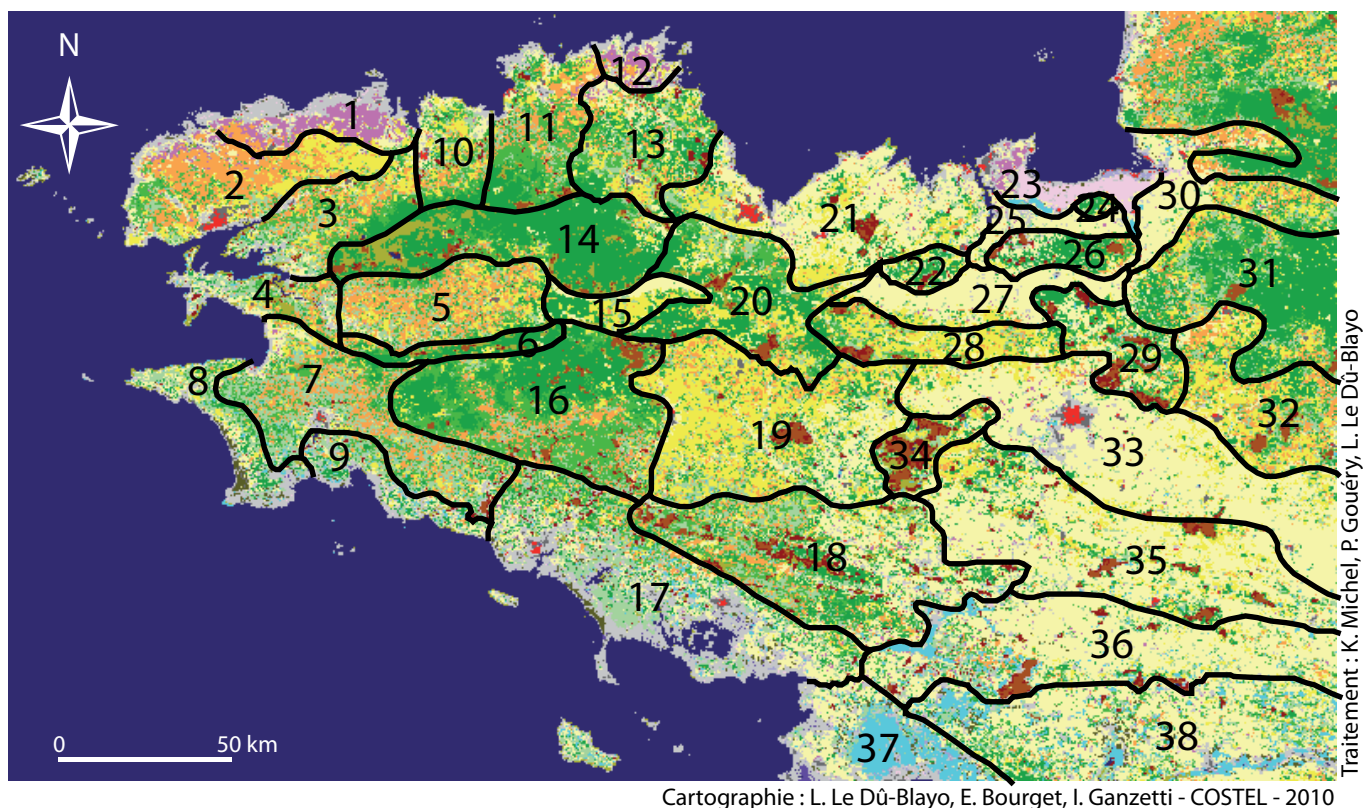

Cartographie : L. Le Dû-Blayo, E. Bourget, I. Ganzetti - COSTEL - 2010

1. Léon légumier

2. Plateau léonard

3. De l'Aulne à l'Elorn

4. Crozon

5. Bassin de Châteaulin

6. Montagnes Noires

7. Cornouaille

8. Baie d'Audierne

9. Cornouaille littorale

10. Trégor morlaisien

11. Trégor

12. Trégor légumier

13. Goëlo

14. Arrée

15. Bassin de St Nicolas du Pelem

16. Cornouaille intérieure

17. Armor morbihanais

18. Reliefs des Landes de Lanvaux

19. Bassin de Pontivy
20. Massif du Mené

21. Plateau du Penthièvre

22. Massif du Hinglé

23. Zone légumière de la baie du Mt St Michel

24. Massif de Saint Broladre

25. Bassin de Pleine-Fougères

26. Massif de St Pierre de Plesguen

27. Bassin d'Evran

28. Collines de Bécherel

29. Collines de Saint Aubin d'Aubigné

30. Avranchin et Val de Sée

31. Bocage mayennais

32. Plateau de Fougères

33. Bassin de Rennes

34. Massif de Brocéliande

35. Plissements de Bain de Bretagne

36. Marches de Bretagne

37. Marais de Guérande et de Brière

38. Loire des promontoires

Figure 8 : Carte des ensembles paysagers en Bretagne

Map of the main landscape unities in Brittany 


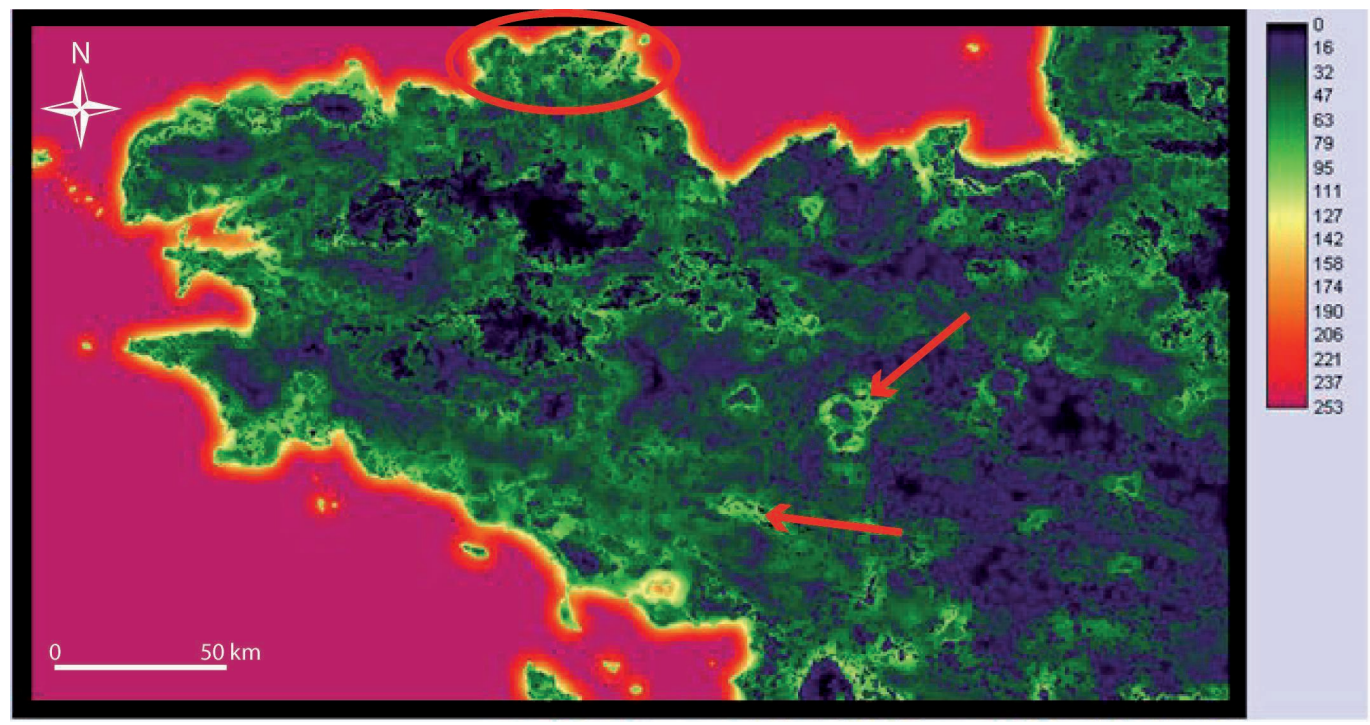

Figure 9 : Carte des distances mathématiques de classement par le logiciel ClaPaS Map of mathematical spaces of ranking by ClaPaS software

\begin{tabular}{|c|c|c|c|}
\hline & Méthode holistique & ClaPaS & Morphologie \\
\hline $\begin{array}{l}\text { Landes de Lanvaux - } \\
\text { Effet de structure }\end{array}$ & $\begin{array}{l}\text { Unités de forme } \\
\text { allongée, en lien } \\
\text { avec le relief et la } \\
\text { géologie }\end{array}$ & $\begin{array}{l}\text { Atténuation de la } \\
\text { forme allongée des } \\
\text { unités paysagères }\end{array}$ & $\begin{array}{l}\text { Morcellement des } \\
\text { UP mais } \\
\text { conservation de la } \\
\text { forme allongée }\end{array}$ \\
\hline $\begin{array}{c}\text { Forêt de Paimpont - } \\
\text { Effet de frontière }\end{array}$ & $\begin{array}{c}\text { Unité } 35 \text { englobant } \\
\text { des paysages autres } \\
\text { que forestiers }\end{array}$ & $\begin{array}{l}\text { Simplification des } \\
\text { paysages de l'Est de } \\
\text { la forêt }\end{array}$ & $\begin{array}{l}\text { Morcellement de } \\
\text { l'unité paysagère } \\
\text { forestière }\end{array}$ \\
\hline $\begin{array}{l}\text { Trégor- } \\
\text { Effet de mixité } \\
\text { paysagère }\end{array}$ & $\begin{array}{l}\text { Une limite } \\
\text { progressive (UP 16), } \\
\text { marque d'un } \\
\text { continuum paysager }\end{array}$ & $\begin{array}{l}\text { Classification en } \\
\text { très petites unités, } \\
\text { avec de nombreux } \\
\text { types sur une petite } \\
\text { zone }\end{array}$ & $\begin{array}{l}\text { Une petite zone non } \\
\text { classée (à l'Est), } \\
\text { preuve de la très } \\
\text { grande mixité du } \\
\text { paysage }\end{array}$ \\
\hline
\end{tabular}

Figure 10 : Comparaison des comportements de différentes méthodes face à divers cas d'incertitude liée aux résultats

results

Comparison of the behaviours of several methods against different cases of uncertainty linked with 
planche XI (Émilie BOURGET, Laurence LE DÛ-BLAYO - Définition d'unités paysagères par télédétection...)

(Marylise COTTET et al. - Mieux comprendre la perception des paysages de bras morts...)

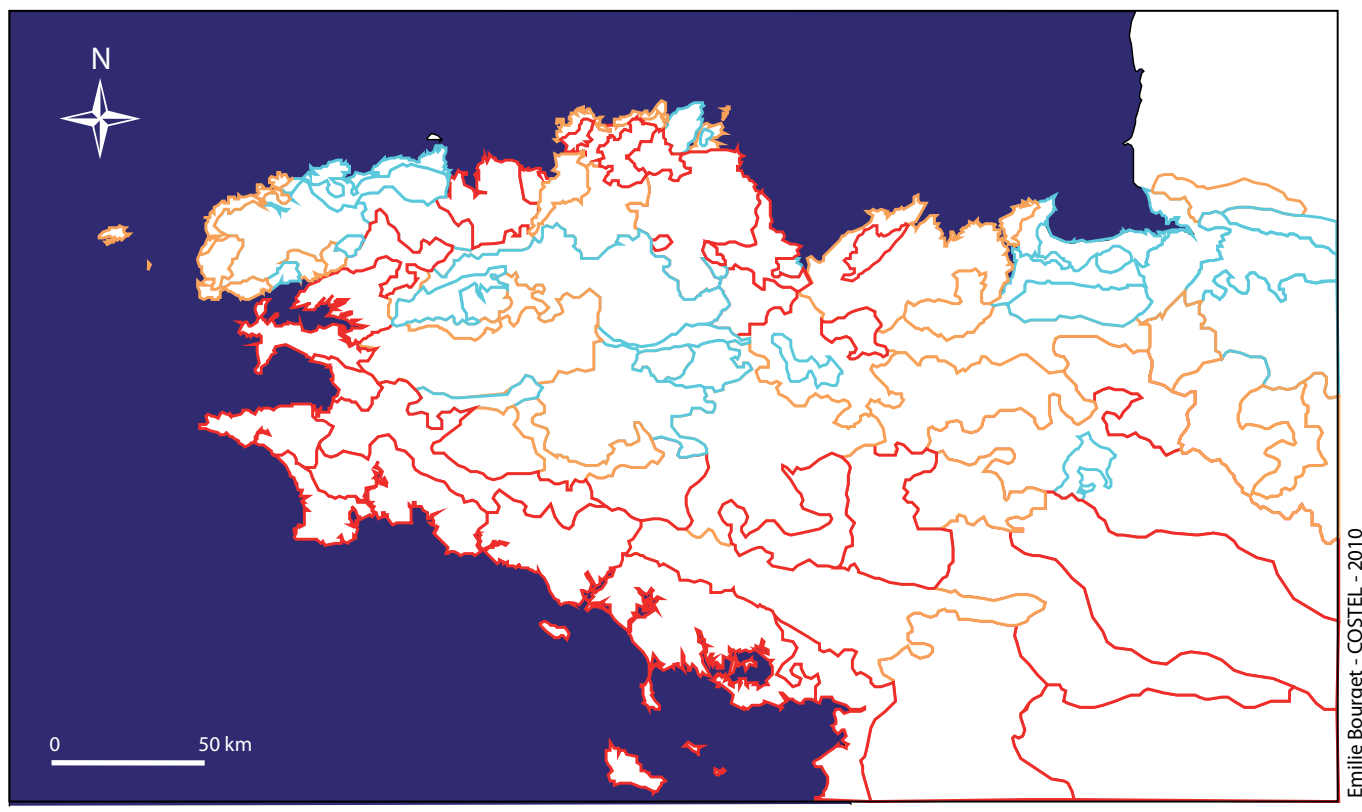

Figure 11 : Typologie de l'incertitude des limites des unités de paysage de synthèse units boundaries

Typology of uncertainty of synthesis landscape

incertitude faible

__ incertitude moyenne incertitude forte
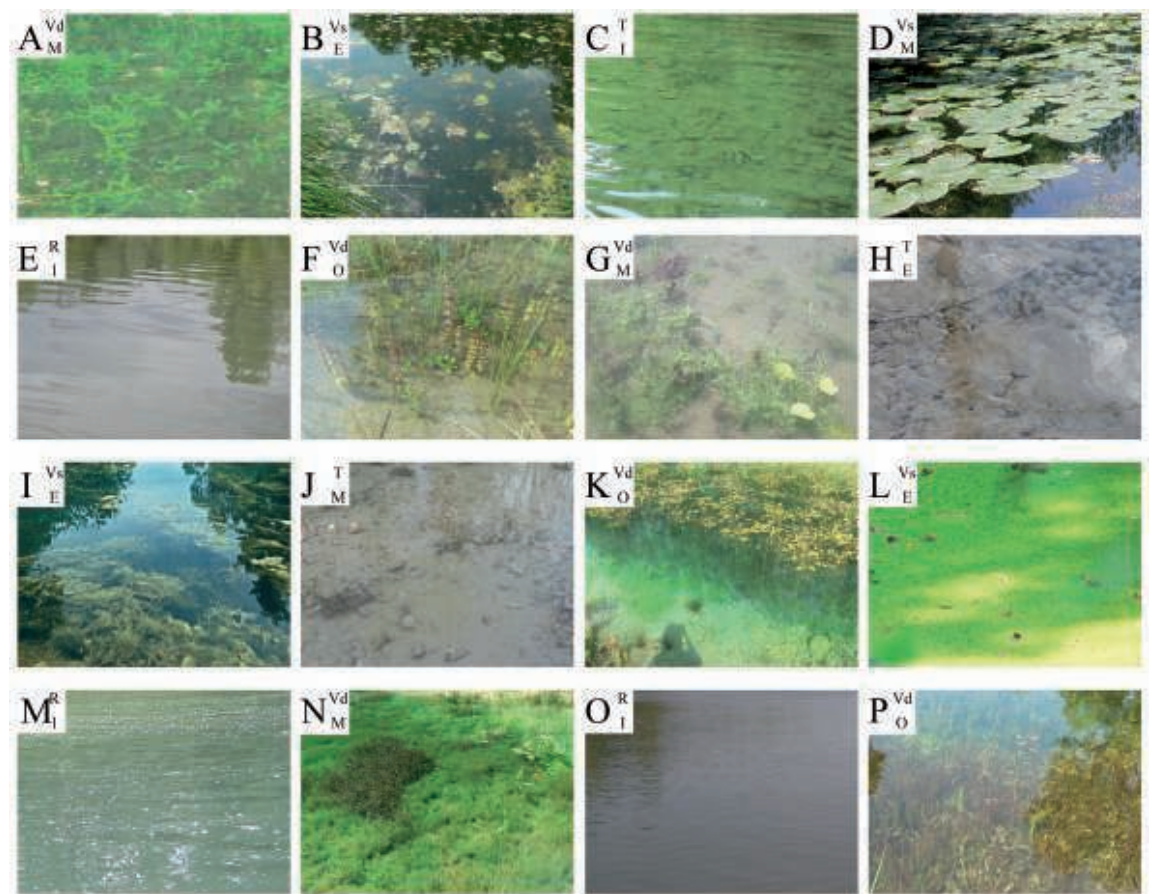

Photo 1 : Photographies des plans d'eau de bras morts utilisées dans le photo-questionnaire Photographs of the floodplain lakes waters used in the photo-questionnaire 